\title{
Network effects in railways
}

\author{
A. Landex \\ Department of Transport, Technical University of Denmark, Denmark
}

\begin{abstract}
Railway operation is often affected by network effects as a change in one part of the network can influence other parts of the network. Network effects occur because the train runs may be quite long and since the railway system has a high degree of interdependencies as trains cannot cross/overtake each other everywhere in the network. First this paper describes network effects in general (section 1). In section 2 the network effects for trains and how they can be measured by scheduled waiting time is described. When the trains are affected by network effects the passengers are also affected. Therefore, sections 3 and 4 describe the network effects for passengers and how they can be measured using passenger delay models. Before the concluding remarks in section 6 , section 5 discusses how the operation can be improved by examining network effects in the planning process.

Keywords: network effect, train delay, passenger delay, scheduled waiting time.
\end{abstract}

\section{Introduction}

When railway capacity and delays are investigated, the analyses are often restricted to a single railway line or section of the network. However, a change in one part of the network can influence other parts of the network. This influence can even be far away from where the original change was made. These influences are denoted as network effects and occur because train routes may be quite long and that the railway system has a high degree of interdependencies as trains cannot cross each other or overtake each other everywhere in the network (see Figure 1).

Network effects are dependent on the given infrastructure and timetable and can result in longer travel times for trains and passengers. The passengers can be further affected due to network effects because not all the wanted correspondences to/from other trains can be kept, due to too many 


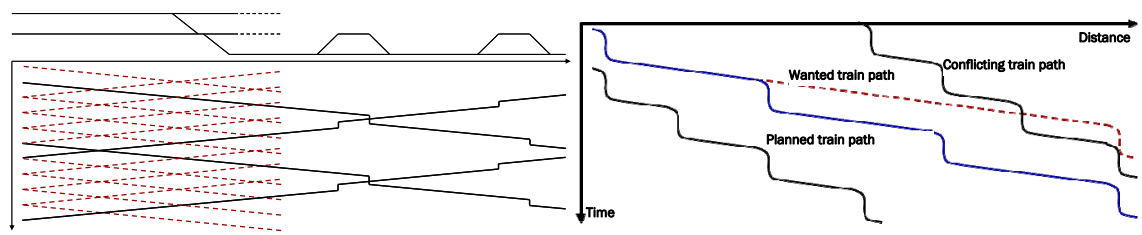

Figure 1: Interdependencies in railway operation.

interdependencies or network effects. Furthermore, the network effects can result in reduced capacity as some trains or train routes can make it impossible to operate other planned/desired trains or train routes.

Identifying network effects of changes on the main lines, a nationwide candidate timetable for one standard hour must be worked out. However, the nationwide timetable in Denmark depends on the train services to/from Germany and Sweden. To evaluate all the network effects it is therefore not enough to create a nationwide candidate timetable. It is necessary to include the trains to/from Germany and Sweden and thereby also the nationwide timetables of Germany and Sweden and so forth.

When the analysis area is large, the risk of network effects is high too. This is because when a large analysis area is examined it can result in bigger changes in the infrastructure and/or timetables. Major changes in the infrastructure and/or timetables may influence many trains in the analysis area, and these trains may influence other trains outside the analysis area.

However, even smaller analysis areas may generate network effects. This is due to the way of planning the timetable in Denmark and many other countries. All train services can be placed in a hierarchy where the train services placed in the top of the hierarchy is planned and timetabled before trains further down in the hierarchy (see Figure 2).

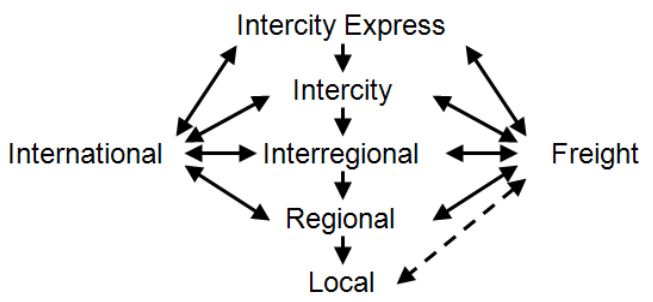

Figure 2: $\quad$ The planning hierarchy [1].

Even small changes in the timetable of a train in the upper level of the hierarchy may influence other trains further down in the hierarchy, because these trains are planned according to the train high up in the hierarchy. Since trains high up in the hierarchy often travel long distances, the changes for other train services can occur far away from the analysis area.

Although the risk of network effects is known many kinds of analysis/projects, the effects of changed timetables and/or infrastructure are only 
studied locally. It can be due to lack of resources, or because the network effects are uncertain (or insignificant), or because one wish only to evaluate the project locally, isolated from the remaining network.

\section{Network effects for trains}

Network effects for trains can be illustrated by scheduled waiting time (SWT). SWT is the difference in running time when comparing a single train on a line with a situation with many trains on the line. SWT on railway lines occurs when the traffic intensity is close to the capacity level due to e.g. mixed operation (slow and fast trains). When close to the capacity level, the operation speeds of fast trains must/will adapt to the slower trains. This will increase the travel time for the trains that under free conditions could run at higher speeds [2].

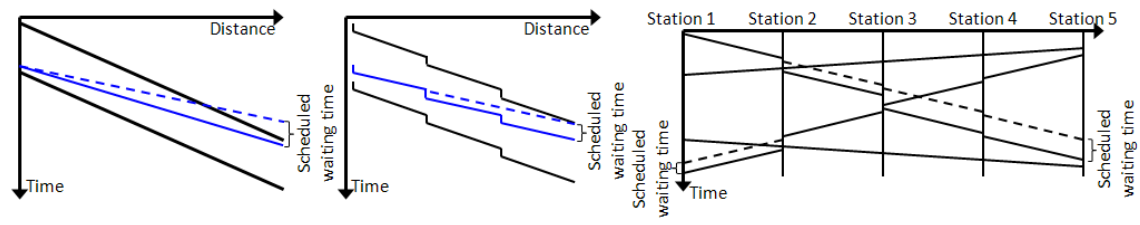

Figure 3: $\quad$ SWT for trains due to other trains in the railway network [3].

To calculate the SWT for trains the Danish developed the SCAN model (Strategic Capacity Analysis of Network) [4, 5] can be used (a similar function is found in the German tool UX-SIMU). SCAN is a computer tool for the calculation of capacity in a railway network. In SCAN capacity is measured as average SWT in a sample of candidate timetables for a given infrastructure alternative.

SCAN can be used in the strategic planning process where the exact infrastructure and timetable is not determined. Therefore, the system is based on a structure where it is only necessary to know the plan of operation (i.e. the number of trains within each category), the infrastructure in a simple way and the main dynamics of the rolling stock [5]. Based on the infrastructure and the plan of operation different timetables are simulated and the SWT is calculated (see figure 4).

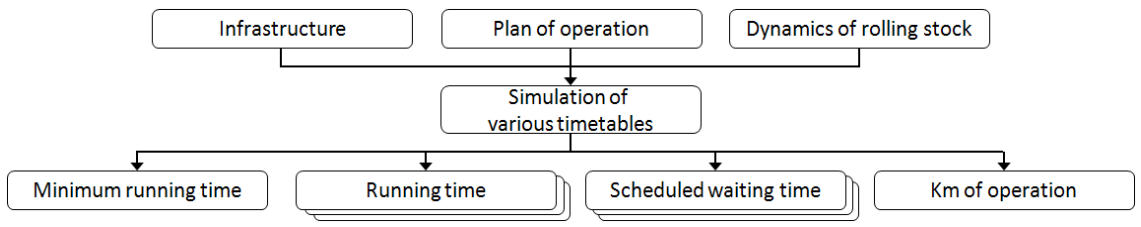

Figure 4: $\quad$ Calculation of SWT $[1,3]$. 
Examining a large number of different timetables based on the same plan of operation will result in different SWT. These different SWT can then be ordered according to the SWT to see the span in SWT. Thereby, it is possible to choose the timetable that has the lowest SWT and still fulfils other potential requirements for the timetable - e.g. possible transfers between trains.

Previous analyses [6-8] have shown that both the size of the network and the connections between trains (correspondences) influences the network effects (see Figure 5).

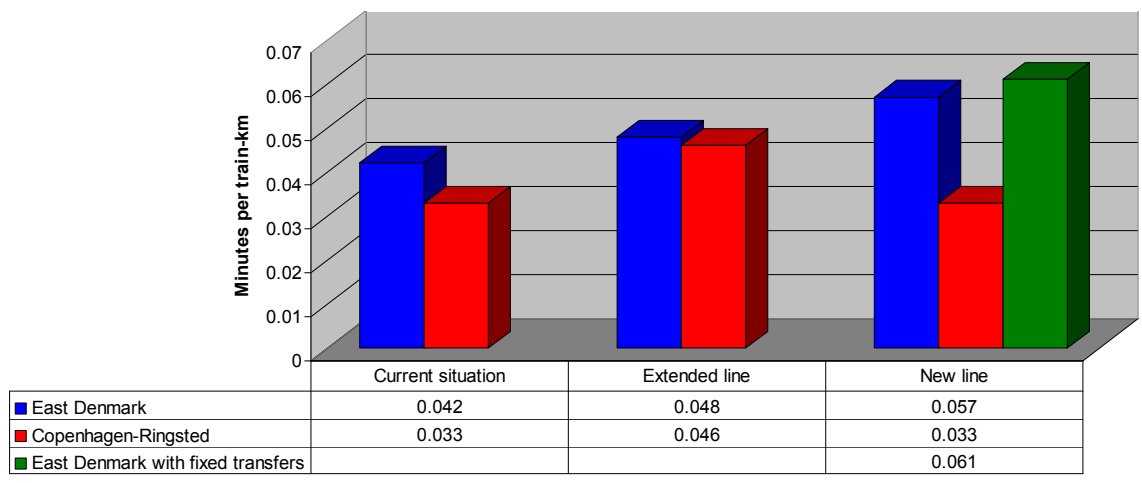

Figure 5: $\quad$ SWT per train km/Network effects. Based on [6-8].

It appears from Figure 5 that the SWT - or network effects - is increasing with the size of the analysis area. Also, transfers between trains (correspondences) increase the SWT. The reason for the increase in SWT is the higher complexity of the operation. Correspondences reduce the degree of freedom in the timetabling which result in a higher risk of SWT. To avoid an increase in SWT; timetable planners have to be more precise when timetabling for larger networks (and networks with correspondences) than for a railway line with no track connection to other railway lines.

\section{Network effects for passengers}

The network effects - or SWT - above is described only for the trains and not the passengers. Network effects for passengers are more complex to calculate than the network effects for the trains. The higher complexity is because the passengers are affected of the networks effects of the trains. Furthermore, passengers often have more options for a journey. The waiting time at the station(s) should also be included in the calculation of network effects for the passengers. To be able to calculate the network effects for the passengers it is necessary to know the timetables - and thereby the network effects for the trains.

As illustrated in section 2 transfers is a network effect. Transfers in public transport networks are unavoidable as it is not possible to design a network where all passengers can travel the direct way from their origin to their destination. It is not all transfers in (larger) public transport networks that will 
have good correspondences as improving one transfer/correspondence might worsen other correspondences.

Figure 6 illustrates a simple railway network with and without correspondence at "Stop B". The travel time between "Stop D" and "Stop C" varies depending on the timetable and thereby the correspondence at "Stop B".

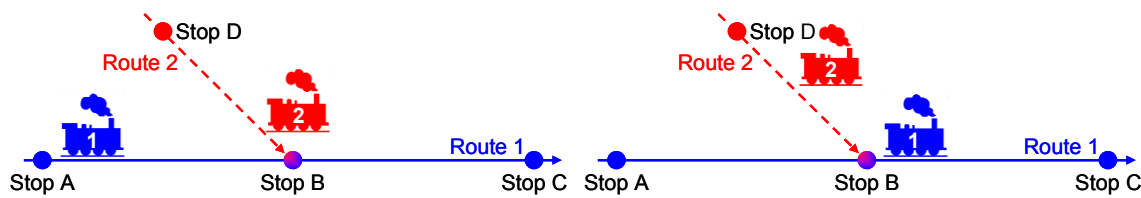

Figure 6: Correspondence between two routes (left) and no correspondence (right) [1].

The example above is straightforward to overview and optimize but for more complex networks the optimization of correspondences becomes complex. Figure 7 shows a journey with two transfers. In the beginning and in the end of the journey there are train routes with 20-minute-frequency but in-between there is a 5-minute-frequency train route. Examining the transfers independently there is good correspondences at both stops but the passengers in the left example in Figure 7 will have no correspondence at the second transfer due to the long waiting time while there is a correspondence on the example to the right in Figure 7.
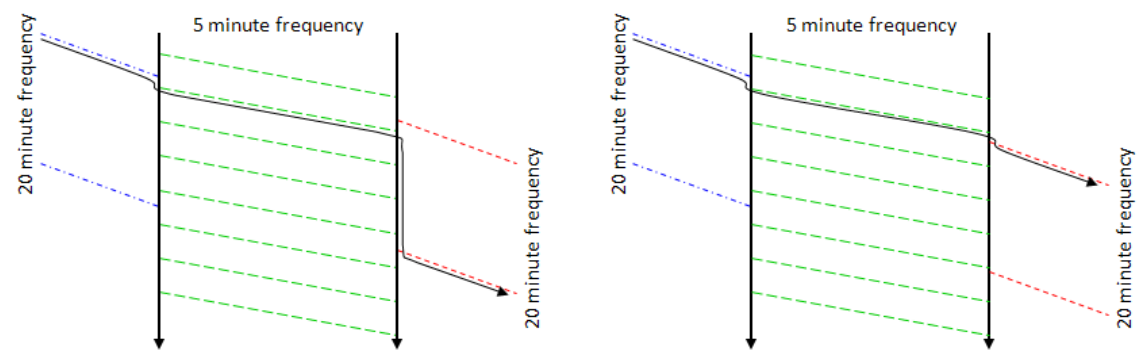

Figure 7: Journey with two transfers: lack of correspondence (left) and correspondence (right) [3].

The network effects of the passengers can due to the dependency on the infrastructure and the timetables be estimated as the (additional) time the passengers spend in the system. This measurement for the network effects is similar to the SWT measurement for the trains.

As the amount of time - or network effects - varies depending on the amount of lost correspondences, the network effects depends on the punctuality of the railway system. To take the punctuality of the railway system into account when calculating the network effects for the passengers it is necessary to simulate the (candidate) timetables. 


\subsection{Calculation of network effects for the passengers}

Network effects for passengers are basically the delays of the passengers compared to the "optimal" timetable. This definition of network effects for the passengers is very similar to the SWT for network effects for the trains. The network effects for passengers should include the trains' risk of delays in the operation too. This is because correspondences are a network effect and that passengers might lose their correspondences if the trains are delayed.

Passenger delays can be calculated in different ways (see Table 1). The simplest way of calculating passenger delays is denoted the $0^{\text {th }}$ generation

Table 1: $\quad$ Methods to calculate passenger delays [9].

\begin{tabular}{|c|c|c|c|c|c|c|c|}
\hline & 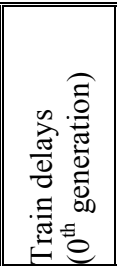 & 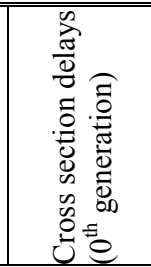 & 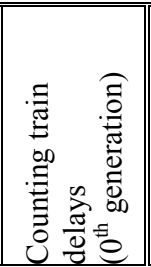 & 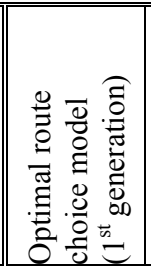 & 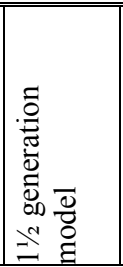 & 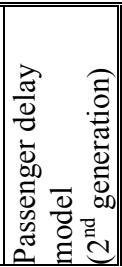 & 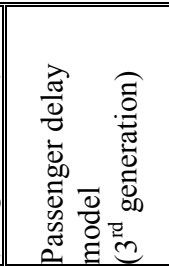 \\
\hline \begin{tabular}{|c|}
$\begin{array}{c}\text { Considerations } \\
\text { of passenger } \\
\text { delays }\end{array}$ \\
\end{tabular} & No & Partly & Partly & Partly & Partly & Yes & Yes \\
\hline $\begin{array}{c}\text { Complexity of } \\
\text { the method } \\
\end{array}$ & \begin{tabular}{|c|} 
Very \\
simple \\
\end{tabular} & Low & Low & Medium & Medium & High & High \\
\hline \begin{tabular}{||c|} 
Needs of \\
information on \\
passenger \\
demand \\
\end{tabular} & No & $\begin{array}{c}\text { Average } \\
\text { aligning } \\
\text { passengers }\end{array}$ & $\begin{array}{c}\text { Counted } \\
\text { passengers }\end{array}$ & $\begin{array}{l}\text { OD } \\
\text { matrix }\end{array}$ & $\begin{array}{l}\text { OD } \\
\text { matrix }\end{array}$ & $\begin{array}{l}\text { OD } \\
\text { matrix }\end{array}$ & OD matrix \\
\hline \begin{tabular}{|c|} 
Passengers \\
may predict \\
delays in the \\
future (full \\
information is \\
assumed) \\
\end{tabular} & No & No & No & Yes & Yes & Partly & $\begin{array}{c}\text { Can be } \\
\text { incorporated }\end{array}$ \\
\hline $\begin{array}{c}\text { Passengers } \\
\text { may arrive } \\
\text { before time if } \\
\text { a better con- } \\
\text { nection } \\
\text { emerge } \\
\end{array}$ & No & No & No & Yes & Yes & Yes & Yes \\
\hline Accuracy & $\begin{array}{l}\text { Very } \\
\text { low }\end{array}$ & Quite low & Fairly low & Low & Medium & Medium & High \\
\hline Bias & $\mid \begin{array}{c}\text { Mostly } \\
\text { under- } \\
\text { estimate } \\
\text { delays }\end{array}$ & $\begin{array}{c}\text { Will quite } \\
\text { often } \\
\text { under- } \\
\text { estimate } \\
\text { delays }\end{array}$ & $\begin{array}{c}\text { Will } \\
\text { fairly } \\
\text { often } \\
\text { under- } \\
\text { estimate } \\
\text { delays } \\
\end{array}$ & \begin{tabular}{||c|} 
Large \\
under- \\
estimation \\
of delays
\end{tabular} & $\begin{array}{c}\text { Under- } \\
\text { estimate } \\
\text { delays }\end{array}$ & $\begin{array}{c}\text { No sys- } \\
\text { tematic } \\
\text { bias }\end{array}$ & $\begin{array}{l}\text { No system- } \\
\text { atic bias }\end{array}$ \\
\hline
\end{tabular}


models. Here the train delays are examined and eventually multiplied with the number of passengers. The $0^{\text {th }}$ generation passenger delay models have the disadvantage that they do not take passengers route choice into account and that the models count passengers who (due to the delays) have reached an earlier train than planned as delayed too.

$1^{\text {st }}, 1 \frac{1 / 2}{2}$ and $2^{\text {nd }}$ generation passenger delay models uses route choice models to estimate the passenger delays. However, the passengers know the delays before they occur. For $2^{\text {nd }}$ generation passenger models the passengers only know the probability of delays based on experience and can plan their route according to that. Using $3^{\text {rd }}$ generation passenger delay models the passengers do not know delays before they occur why they cannot react on the delays before they know about them.

Estimating the network effects of the passengers without delays $1^{\text {st }}$ generation models and above can be used. Thus all these models can calculate the time the passengers spend in the railway network. However, $3^{\text {rd }}$ generation models are the best if the network effects should be calculated in case of train delays or if sensitivity analyses have to be carried out. As $3^{\text {rd }}$ generation models require the same work effort as $1^{\text {st }}, 1 \frac{1}{2}$ and $2^{\text {nd }}$ generation models it is recommended that $3^{\text {rd }}$ generation models are used as it is possible to extend the analyses in the future.

\subsection{1 $3^{\text {rd }}$ generation passenger delay models}

$3^{\text {rd }}$ generation models assume that passengers are planning their optimal desired route according to the official timetable (or by incorporating expected delays using a $2^{\text {nd }}$ generation model). However, if delays occur over a certain threshold during the trip, the passengers are assumed to reconsider the route at that point in time and space along the route. If a train is completely cancelled, the passengers reconsider their choice without a threshold.

The main benefit of $3^{\text {rd }}$ generation models is that it is more realistic and precise than the prior generations of passenger delay models. The disadvantage is that it is more complicated to implement, and that the calculation time is larger. This is because the route choice model has to be re-run at the point in time and space where the schedule is delayed.

A core assumption is that the paths the passengers choose in the a priori path choice strategy are stored as a sequence of lines (each with a specific run) and transfer stations. The passengers are then assumed to try to follow the same sequence of transfer stations and lines as planned, but the passengers may use different train runs for each line. The difference in passenger's time between first and the second route choice assignment equals the passenger's delays.

\section{Calculating network effects of passengers}

As described in section 2 it is relatively straightforward to calculate the network effects for trains. However, cases with small network effects for trains do not necessarily result in small network effects for passengers - and vice versa.

Although the SCAN model calculates the network effects for trains in terms of SWT [5], it can be used as an input to calculate the network effects for 
passengers too. This is because the output timetables of SCAN can be used together with a route choice model to calculate the passenger's time usage in the railway network in case of no delays. In this way the network effects for the passengers can be determined as the difference between the times used in the actual analyzed timetable and the best analyzed timetable.

A problem with the modelling approach described above is that the SCAN model does not use time supplements why the model only can be used to evaluate the plan of operation. Alternatively, the North American Train Performance Calculator (TPC) [10] can be used instead to generate a large amount of timetables which can be investigated. However, the TPC model is developed for North American conditions where there is no regular timetable the trains are operated more or less improvised [11]. While including time supplements in the SCAN model and/or adapting the TPC model for regular timetables the methodology is well suited for strategic analyzes in the Danish/European content.

Simulation models based on future plans of operation are well suited for strategic analyzes but it is difficult to examine where the problems are most severe. Therefore, it is difficult to examine where the infrastructure should be improved and the effect of the improvement. Furthermore, the strategic analyzes do not take (risk of) delays into account why the results do not reflect the actual operation.

To reflect the actual operation and to be able to examine problems in the infrastructure, "traditional" simulation is necessary. Therefore, it is necessary to build up the infrastructure and timetables before simulating the operation in case of disturbances and then evaluate the results for both trains and passengers (see Figure 8).

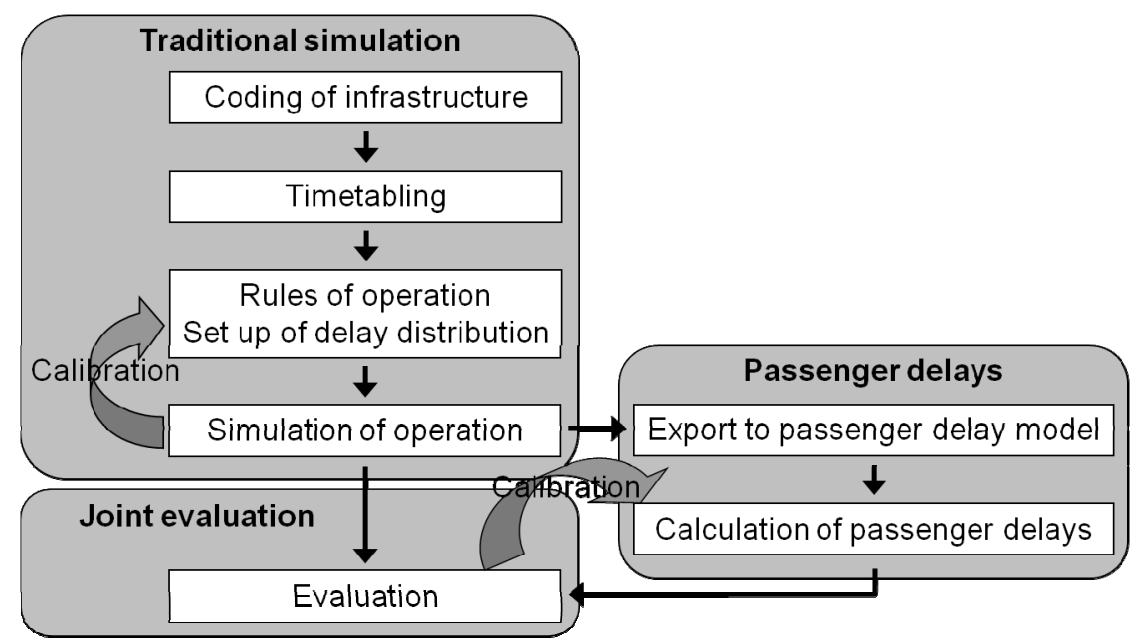

Figure 8: $\quad$ Traditional simulation of railway traffic with passenger delays [1]. 
The different infrastructures and timetables will result in different amount of time for the passengers. These different amounts of time can then be compared used to evaluate the network effects of the passengers. However, traditional simulation projects are time consuming but by combining microscopic and macroscopic models - so-called meso models - (as e.g. done by Railnet Austria [12]) can reduce the workload of simulations.

\section{Discussion}

Network effects of passengers can be used to improve the timetables for the passengers. This can be done by comparing timetables from different years and evaluate different travel relations together with the total time spend in the railway system.

It is not only possible to evaluate previous and present timetables. By examining different candidate timetables it is possible to examine the network effects of future timetables. In this way different timetable strategies can be examined - e.g. an additional overtaking. When examining the network effects of an additional overtaking it is possible to evaluate both the time gain for the passengers in the fast train and the time loss for the passengers in the train that is overtaken. This examination can either be done locally for a single railway line or for the entire system including transfers to/from other trains.

Improving the timetables not taking the risk of delays into account can result in a too optimized timetable where even small delays will result in lost correspondences for the passengers etc. To take common delays into account simulation of the timetables with a typical delay distribution can be performed. The time for the passengers - or the network effects - can then be calculated based on the simulated timetables. In this way it is possible to optimize the timetables for the passengers and make the timetables robust for network effects and future delays.

Calculation of network effects for passengers can improve the operation. This is because network effects for passengers can be taken into account when planning for contingency operation. When the troubled operation then occurs and the timetable for contingency operation is taken into operation the network effects of the passengers will be taken into account implicitly.

\section{Conclusion}

Railway operation is often affected by network effects as a change in one part of the network can influence other parts of the network. This influence can even be far away from where the original change was made. The network effects occur because the train routes (often) are quite long and that the railway system has a high degree of interdependencies as trains cannot cross/overtake each other everywhere in the network.

Network effects can affect both trains and passengers. Network effects for trains can be measured by SWT for the trains while the network effects for the passengers can be measured as passenger delays compared to the optimal 
timetable. It is more complex to calculate network effects for passengers as the network effects for the passengers depend on the network effects for the trains. Moreover, delays in the operation can enlarge the network effects for the passengers as correspondences might be lost.

This paper suggests methods to calculate network effects for trains and passengers. Using these methods to calculate network effects for different candidate timetables it is to test different timetable strategies and choose the best strategy for the final timetable. In this way it is possible to improve the timetables for both the operator(s) and the passengers.

\section{References}

[1] Landex, A., Methods to estimate railway capacity and passenger delays. PhD thesis, Technical University of Denmark, 2008.

[2] Salling, K.B. and Landex, A., Computer based ex-ante evaluation of the planned railway line between Copenhagen and Ringsted by use of a new Decision Support System named COSIMA-DSS. Comp Rail X, eds. J. Allen, C.A. Brebbia, A.F. Rumsey, G. Sciutto, S. Sone and C.J. Goodman, WIT press, p. 65, 2006.

[3] Landex, A., Computation and Evaluation of Scheduled Waiting Time for Railway Networks. Comp Rail XII, WIT press, p. 911, 2010.

[4] Kaas, A.H., Methods to calculate capacity of railways, $\mathrm{PhD}$ thesis, Technical University of Denmark, 1998.

[5] Kaas, A.H., Development and practical use of a capacity model for railway networks, Structural Integrity and Passenger Safety, ed. C.A. Brebbia, WIT press, p. 73, 1998.

[6] Hansen, S., Landex, A. and Kaas, A.H., The network effects of railway investments, Comp Rail X, eds. J. Allen, C.A. Brebbia, A.F. Rumsey, G. Sciutto, S. Sone and C.J. Goodman, WIT press, pp. 45, 2006.

[7] Hansen, S., Large Transport Infrastructure Investments and their Strategic Impacts with a Special Focus on Enterprises, $\mathrm{PhD}$ thesis, Technical University of Denmark, 2004.

[8] Landex, A., Kaas, A.H. and Hansen, S., Railway Operation, Technical University of Denmark, 2006.

[9] Nielsen, O.A., Landex, A. and Frederiksen, R.D., Passenger Delay Models for Rail Networks $2^{\text {nd }}$ workshop on the Schedule-based approach in Dynamic Transit Modelling (SBDTM 2005), Kluwer Academic, 2005.

[10] White, T.A., The development and use of dynamic traffic management simulation in North America, $2^{\text {nd }}$ International Seminar on Railway Operations Research, eds. I.A. Hansen, A. Radtke, J.P. Pachl and E. Wendler, 2007.

[11] White, T.A., North American Experience with Timetable-Free Railway Operation, $1^{\text {st }}$ International Seminar on Railway Operations Modelling and Analysis, eds. I.A. Hansen, F.M. Dekking, R.M.P. Goverde, B. Heidergott and L.E. Meester, 2005. 
[12] Sewcyk, B., Radtke, A. and Wilfinger, G., Combining Microscopic and Macroscopic Infrastructure Planning Models, $2^{\text {nd }}$ International Seminar on Railway Operations Modeling and Analysis, eds. I.A. Hansen, A. Radtke, J.P. Pachl and E. Wendler, 2007. 